\title{
A MOLECULAR KINETIC THEORY OF INHOMOGENEOUS LIQUID CRYSTAL FLOW AND THE SMALL DEBORAH NUMBER LIMIT*
}

\author{
WEINAN $\mathrm{E}^{\dagger}$ AND PINGWEN ZHANG
}

Dedicated to Professor Björn Engquist on the occasion of his sixtieth birthday

\begin{abstract}
The Doi kinetic theory for homogeneous flows of rodlike liquid crystalline polymers (LCPs) is extended to inhomogeneous flows through introducing a nonlocal intermolecular potential. An extra term in the form of an elastic body force comes out as a result of this extension. Systematic asympototic analysis in the small Deborah number limit is carried out, and the classical EricksenLeslie equations are derived in this limit. The Leslie coefficients are derived in terms of molecular parameters, and the Ericksen stress emerges from the body force.
\end{abstract}

Key words. Rodlike liquid crystal polymers, Doi equation, Ericksen-Leslie theory, Inhomogeneous flow, Body force

AMS subject classifications. 76B03,65M12,35Q35

1. Introduction. The Doi kinetic theory for spatially homogeneous flows of rodlike molecules have been very successful in describing the properties of liquid crystal polymers in a solvent [2]. This theory models the orientational distribution function of the liquid crystal polymers and takes into account the effects of hydrodynamic flow, Brownian motion and intermolecular forces on the molecular orientation distribution. However, it does not include effects such as distortional elasticity. Therefore it is valid only in the limit of spatially homogeneous flows.

On the other hand, for small molecule liquid crystals, distortional elasticity is described quite well by the classical Frank energy. For hydrodynamics, the EricksenLeslie theory is also quite adequate except for disclinations.

Attempts have been made to either extend the Ericksen-Leslie theory to flows in the presence of defects such as disclinations, or to extend the Doi theory to inhomogeneous flows. Ericksen [3] introduced an order parameter that describes the local degree of orientation. Tsuji and Rey $[16,17]$ added distortional elasticity via the Landau- de Gennes free energy to the kinetic equation of the Doi theory but did not give a stress tensor. All these approaches are phenomenological in nature, and the resulting theories are unsatisfactory in one way or another. First, they invariably contain a large number of unknown parameters which in general are difficult to determine using experimental results. This causes both conceptual and practical difficulties when dealing with spatial distortions, especially the severe distortions near defects. Secondly, the use of the Landau-de Gennes expansion is unjustified in these theories. A third drawback of the phenomenological theories is the lack of consistency with existing theories and among themselves. We believe that a molecular approach would be more advantageous than a phenomenological one.

A molecular theory for distortional elasticity was put forward by Marrucci and Greco [13] who proposed a nonlocal mean field nematic potential for LCPs which accounts for spatial variations in the molecular orientation distribution. This is done by

\footnotetext{
*Received December 1, 2005; accepted for publication August 2, 2006.

${ }^{\dagger}$ Department of Mathematics and PACM, Princeton University, NJ 08544, USA (weinan@math. princeton.edu).

${ }_{\ddagger}^{\ddagger}$ LMAM and School of Mathematical Sciences, Peking University, Beijing 100871, P.R. China (pzhang@pku.edu.cn).
} 
resurrecting the tensorial order parameter notion and adding a square gradient term of this tensorial order parameter in the free energy. Feng et. al. [6] derived a molecularly based constitutive theory for nematic LCPs which incorporates distortional elasticity via the Marrucci-Greco potential. In the limit of weak flows and small distortions, they demonstrated that the theory properly reduces to the Ericksen-Leslie theory.

As an extension of the Kuzuu-Doi [8] theory to hydrodynamics of inhomogeneous liquid crystalline polymers, Wang [20] models the LCP molecules as spheroids of equal shape and sizes, he derives an intermolecular potential which could be considered as an extension of the Marrucci-Greco potential.

We intend to develop a theory for inhomogeneous flows of liquid crystalline polymers with few adjustable parameters that could model a variety of configurations and dynamics of polymeric liquid crystal systems. In the present paper, we will follow the set-up initiated in [21]. We will give a quick review of that framework and then we will focus on the problem of studying the asymptotic limit as the Deborah number goes to zero. Our purpose is to derive the well-known Ericksen-Leslie equations from systematic asymptotic analysis. Some initial ideas of this work can be found in [9].

2. Kinetic molecular theory for liquid crystal polymer flow. Our starting point for the derivation of the Ericksen-Leslie equation is a molecular theory in the form of a kinetic equation describing the position-orientation distribution function. The original idea of modeling liquid crystals using the orientation distribution function goes back to Onsager [15]. Onsager was only concerned with the isotropicnematic phase transition, and did not include effects of fluid dynamics. Doi extended Onsager's theory to the case of homogeneous flows [2]. Inhomogeneous flows were first studied in this setting by Marrucci and Greco [13], and subsequently by many people $[6,20]$. Instead of using the distribution as the sole order parameter, they used a combination of the tensorial order parameter and the distribution function, and used the spatial gradients of the tensorial order parameter to describe the spatial variations. This is a departure from the original motivation that led us to the kinetic theory. Therefore it is still desirable to set up a formalism in which the interaction between molecules are treated more directly using the position-orientation distribution function via interaction potentials, and this was the motivation of the work of Wang, E, Liu and Zhang [21]. Here we will give a quick review of that formalism.

2.1. The intermolecular potentials. We model the LCP molecules as rigid rods of equal size. Let $\Omega$ be a material volume in which the solution of LCPs resides and let $f(\mathbf{x}, \mathbf{m}, t)$ be the number density function (NDF) in place of the probability density function (PDF) for the number of molecules parallel to the $\mathbf{m}$ direction at material point $\mathbf{x}$ and time $t$. We extend the free energy in the Doi kinetic theory to include effects of nonlocal intermolecular interactions through an interaction potential as follows:

$A(f)=k_{B} T \int_{\Omega} \int_{\|\mathbf{m}\|=1}\left[f(\mathbf{x}, \mathbf{m}, t) \ln f(\mathbf{x}, \mathbf{m}, t)-f(\mathbf{x}, \mathbf{m}, t)+\frac{1}{2 k_{B} T} U(\mathbf{x}, \mathbf{m}, t) f(\mathbf{x}, \mathbf{m}, t)\right] d \mathbf{m} d \mathbf{x}$,

where $k_{B}$ is the Boltzmann constant and $T$ is the absolute temperature. The meanfield intermolecular potential $U(\mathbf{x}, \mathbf{m}, t)$ is defined by

$$
U(\mathbf{x}, \mathbf{m}, t)=k_{B} T \int_{\Omega} \int_{\|\mathbf{m}\|=1} B\left(\mathbf{x}, \mathbf{x}^{\prime} ; \mathbf{m}, \mathbf{m}^{\prime}\right) f\left(\mathbf{x}^{\prime}, \mathbf{m}^{\prime}, t\right) d \mathbf{m}^{\prime} d \mathbf{x}^{\prime} .
$$


Here $B\left(\mathbf{x}, \mathbf{x}^{\prime} ; \mathbf{m}, \mathbf{m}^{\prime}\right)$ is the interaction kernel. It should be symmetric with respect to the interchange of $\mathbf{m}$ and $\mathbf{m}^{\prime}, \mathbf{x}$ and $\mathbf{x}^{\prime} . B$ is often translation invaraint and hence it can be written in the form

$$
B\left(\mathbf{x}-\mathbf{x}^{\prime} ; \mathbf{m}, \mathbf{m}^{\prime}\right) .
$$

Examples of $B$ include the Onsager potential

$$
B\left(\mathbf{x}-\mathbf{x}^{\prime} ; \mathbf{m}, \mathbf{m}^{\prime}\right)=\alpha\left|\mathbf{m} \times \mathbf{m}^{\prime}\right|
$$

and the Maier-Saupe potential

$$
B\left(\mathbf{x}-\mathbf{x}^{\prime} ; \mathbf{m}, \mathbf{m}^{\prime}\right)=\alpha\left|\mathbf{m} \times \mathbf{m}^{\prime}\right|^{2}
$$

describing excluded volume effects. For spheroidal molecules, the excluded volume with finite range molecular interaction was used by Wang [20]. We suggest a simple potential

$$
B\left(\mathbf{x}-\mathbf{x}^{\prime} ; \mathbf{m}, \mathbf{m}^{\prime}\right)=\alpha\left|\mathbf{m} \times \mathbf{m}^{\prime}\right|^{2} \frac{1}{\varepsilon^{d}} g\left(\frac{\mathbf{x}-\mathbf{x}^{\prime}}{\varepsilon}\right),
$$

where $d$ is the spatial dimension, $g$ is an approximate $\delta$-function that satisfies the condition $g \geq 0, \int_{-\infty}^{\infty} g(r) d r=1, g(r)=0$ if $|r| \geq 1$. This potential neglects the interaction between orientation and position. But it is sufficient in many cases.

The number density of the LCP at a material point $\mathbf{x}$ is given by

$$
\rho(\mathbf{x}, t)=\int_{\|\mathbf{m}\|=1} f(\mathbf{x}, \mathbf{m}, t) d \mathbf{m} .
$$

The chemical potential is given by

$$
\mu=\frac{\delta A}{\delta f}
$$

a simple calculation leads to

$$
\mu=k_{B} T \ln f(\mathbf{x}, \mathbf{m}, t)+U(\mathbf{x}, \mathbf{m}, t) .
$$

2.2. Smoluchowski equation. We will treat the LCP system as being incompressible. Accounting for effects of both the translational and rotational diffusion as well as convection and following the derivation given in [2], we obtain the Smoluchowski equation for the LCP system as follows:

$$
\begin{aligned}
\frac{d f}{d t}= & \frac{1}{k_{B} T} \nabla \cdot\left\{\left[D_{\|} \mathbf{m m}+D_{\perp}(I-\mathbf{m m})\right] \cdot(\nabla \mu) f\right\} \\
& +\frac{D_{r}}{k_{B} T} \mathcal{R} \cdot(f \mathcal{R} \mu)-\mathcal{R}(\mathbf{m} \times \kappa \cdot \mathbf{m} f),
\end{aligned}
$$

where $D_{\|} \geq 0$ and $D_{\perp} \geq 0$ are respectively the translational diffusion coefficients parallel and normal to the orientation of the LCP molecule, $D_{r}=\frac{\xi_{r}}{k_{B} T}$ is the rotary diffusivity, $\nabla$ is the gradient operator with respect to the spatial variable $\mathbf{x}, \nabla_{\mathbf{m}}$ is the gradient operator with respect to the orientational variable $\mathbf{m}, \mathcal{R}=\mathbf{m} \times \nabla_{\mathbf{m}}$ is the rotational gradient operator. $\kappa=(\nabla \mathbf{v})^{T}$ is the velocity gradient tensor, and $\frac{d}{d t}$ 
is the material derivative $\frac{\partial}{\partial t}+\mathbf{v} \cdot \nabla$. In (2.6) the first and second term at the right hand side model respectively the translational and rotational diffusion of the LCPs, the last term models the distortion caused by the velocity gradients.

Let $L_{0}$ be the typical size of the flow region, $V_{0}$ be the typical velocity scale, $T_{0}=\frac{L_{0}}{V_{0}}$ be a typical convective time scale. Another important time scale is the relaxational time scale due to orientation diffusion: $T_{r}=\frac{\xi_{r}}{k_{B} T}$. The ratio of these two time scales is an important parameter called the Deborah number

$$
D e=\frac{T_{r}}{T_{0}}=\frac{\frac{\xi_{r}}{k_{B} T}}{\frac{L_{0}}{V_{0}}}=\frac{\xi_{r} V_{0}}{k_{B} T L_{0}} .
$$

Let

$$
\varepsilon=\frac{L}{L_{0}}
$$

where $L$ is the length of the rods. We can then non-dimensionalize the kinetic equation, and obtain

$$
\begin{aligned}
\frac{\partial f}{\partial t}+\nabla \cdot(\mathbf{v} f)= & \frac{\varepsilon^{2}}{D e} \nabla \cdot\left\{\left[D_{\|}^{*} \mathbf{m m}+D_{\perp}^{*}(I-\mathbf{m m})\right] \cdot(\nabla f+f \nabla \tilde{U})\right\} \\
& +\frac{1}{D e} \mathcal{R} \cdot(\mathcal{R} f+f \mathcal{R} \tilde{U})-\mathcal{R} \cdot(\mathbf{m} \times \kappa \cdot \mathbf{m} f), \\
\tilde{U}= & \int_{\Omega} \int_{\|\mathbf{m}\|=1} B\left(\mathbf{x}, \mathbf{x}^{\prime} ; \mathbf{m}, \mathbf{m}^{\prime}\right) f\left(\mathbf{x}^{\prime}, \mathbf{m}^{\prime}, t\right) d \mathbf{m}^{\prime} d \mathbf{x}^{\prime} .
\end{aligned}
$$

where

$$
D_{\|}^{*}=\frac{L_{0} D e}{V_{0} L^{2}} D_{\|}
$$

and

$$
D_{\perp}^{*}=\frac{L_{0} D e}{V_{0} L^{2}} D_{\perp}
$$

are the respectively the non-dimensionalized translational diffusion coefficients parallel and normal to the orientation of the LCP molecule.

2.3. Constitutive equation for the the stress tensor. Now consider liquid crystal polymer flows, the total stress is the sum of the viscous stress $\tau^{s}$ and the elastic stress $\tau^{e}$,

$$
\tau=\tau^{s}+\tau^{e} .
$$

There are two contributions to the viscous stress, one from the solvent and the other from the constraint force arising from the rigidity of the rod, derived in [2]

$$
\tau^{s}=2 \eta_{s} \mathbf{D}+\frac{1}{2} \xi_{r} \mathbf{D}:\langle\mathbf{m m m m}\rangle,
$$

where $\mathbf{D}=\frac{1}{2}\left(\kappa+\kappa^{T}\right)=\frac{1}{2}\left(\nabla \mathbf{v}+(\nabla \mathbf{v})^{T}\right)$ is the strain rate tensor, $\eta_{s}$ is the solvent viscosity, and

$$
\langle(\cdot)\rangle=\int_{\|\mathbf{m}\|=1}(\cdot) f(\mathbf{x}, \mathbf{m}, t) d \mathbf{m}
$$


is the ensemble average with respect to the $\operatorname{NDF} f(\mathbf{x}, \mathbf{m}, t)$.

The elastic stress is derived through a generalized virtual work principle [2]. Consider an infinitesimal displacement given by $\delta \mathbf{u}=\mathbf{v} \delta t$, corresponding to a deformation rate $\delta \epsilon=\nabla \mathbf{v} \delta t$. The variation of the free energy over the control volume $\Omega$ in response to the infinitesimal deformation and displacement can be expressed as:

$$
\delta A=\int_{\Omega}\left(\delta \epsilon: \tau^{e}-\delta \mathbf{u} \cdot \mathbf{F}^{e}\right) d \mathbf{x}
$$

where $\mathbf{F}^{e}$ is the body force and $\tau^{e}$ is the elastic stress.

$$
\mathbf{F}^{e}=-\langle\nabla \mu\rangle
$$

Therefore we identify the elastic stress as

$$
\begin{aligned}
\tau^{e} & =-\langle\mathbf{m m} \times \mathcal{R} \mu\rangle \\
& =3 k_{B} T S_{\alpha \beta}-\left\langle m_{\alpha}(\mathbf{m} \times \mathcal{R} U)_{\beta}\right\rangle .
\end{aligned}
$$

where

$$
S_{\alpha \beta}=\left\langle m_{\alpha} m_{\beta}-\frac{1}{3} \delta_{\alpha \beta}\right\rangle
$$

Since

$$
\delta f=\frac{d f}{d t} \delta t=-\mathcal{R} \cdot(\mathbf{m} \times \kappa \cdot \mathbf{m} f) \delta t .
$$

we have

$$
\begin{aligned}
\delta A & =k_{B} T \int_{\Omega} \int_{\|\mathbf{m}\|=1}\left[\left(\ln f+\frac{1}{k_{B} T} U\right) \delta f+\frac{1}{2 k_{B} T}(\delta U f-U \delta f)\right] d \mathbf{m} d \mathbf{x} \\
& =k_{B} T \int_{\Omega} \int_{\|\mathbf{m}\|=1}\left[\mu \frac{d f}{d t} \delta t+\frac{1}{2 k_{B} T}(\delta U f-U \delta f)\right] d \mathbf{m} d \mathbf{x} .
\end{aligned}
$$

The first term on the right hand side can be written as

$$
\begin{aligned}
\int_{\Omega} \int_{\|\mathbf{m}\|=1} \mu \frac{d f}{d t} \delta t d \mathbf{m} d \mathbf{x} & =\int_{\Omega} \int_{\|\mathbf{m}\|=1} \mu[-\mathcal{R} \cdot(\mathbf{m} \times \kappa \cdot \mathbf{m} f)] \delta t d \mathbf{m} d \mathbf{x} \\
& =\int_{\Omega} \int_{\|\mathbf{m}\|=1} \mathcal{R} \mu \cdot(\mathbf{m} \times \kappa \cdot \mathbf{m}) f \delta t d \mathbf{m} d \mathbf{x} . \\
& =-\int_{\Omega} \int_{\|\mathbf{m}\|=1}(\mathbf{m} \times \mathcal{R} \mu \mathbf{m}): \kappa \delta t f d \mathbf{m} d \mathbf{x}
\end{aligned}
$$

Hence the elastic stress can be identified as

$$
\tau^{e}=-\langle\mathbf{m m} \times \mathcal{R} \mu\rangle .
$$


The second part of the right hand side reads

$$
\begin{aligned}
& \int_{\Omega} \int_{\|\mathbf{m}\|=1} \frac{1}{2}(\delta U f-U \delta f) d \mathbf{m} d \mathbf{x} \\
= & \int_{\Omega} \int_{\|\mathbf{m}\|=1} \frac{1}{2}\left(\frac{d U}{d t} f-U \frac{d f}{d t}\right) \delta t d \mathbf{m} d \mathbf{x} \\
= & \frac{k_{B} T}{2} \int_{\Omega} d \mathbf{x} \int_{\|\mathbf{m}\|=1} d \mathbf{m} \int_{\left\|\mathbf{m}^{\prime}\right\|=1} d \mathbf{m}^{\prime} \int_{\Omega} d \mathbf{x}^{\prime} f(\mathbf{x}, \mathbf{m}, t) \\
& {\left[\left(\mathbf{v}(\mathbf{x}, t)-\mathbf{v}\left(\mathbf{x}^{\prime}, t\right)\right) \cdot \nabla B\left(\mathbf{x}-\mathbf{x}^{\prime} ; \mathbf{m}, \mathbf{m}^{\prime}\right) f\left(\mathbf{x}^{\prime}, \mathbf{m}^{\prime}, t\right) \delta t\right.} \\
& \left.+B\left(\mathbf{x}-\mathbf{x}^{\prime} ; \mathbf{m}, \mathbf{m}^{\prime}\right) \frac{d f}{d t}\left(\mathbf{x}^{\prime}, \mathbf{m}^{\prime}, t\right) \delta t\right] \\
& -\frac{1}{2} \int_{\Omega} \int_{\|\mathbf{m}\|=1} U(\mathbf{x}, \mathbf{m}, t) \frac{d f(\mathbf{x}, \mathbf{m}, t)}{d t} \delta t d \mathbf{m} d \mathbf{x} \\
= & \frac{1}{2} \int_{\Omega} \int_{\|\mathbf{m}\|=1} f(\mathbf{x}, \mathbf{m}, t) \mathbf{v}(\mathbf{x}, t) \cdot \nabla U \delta t d \mathbf{m} d \mathbf{x} \\
& -\frac{k_{B} T}{2} \int_{\Omega} d \mathbf{x} \int_{\|\mathbf{m}\|=1} d \mathbf{m} \int_{\left\|\mathbf{m}^{\prime}\right\|=1} d \mathbf{m}{ }_{\Omega}^{\prime} d \mathbf{x}^{\prime} f\left(\mathbf{x}^{\prime}, \mathbf{m}^{\prime}, t\right) \\
& \mathbf{v}(\mathbf{x}, t) \cdot \nabla_{\mathbf{x}^{\prime}} B\left(\mathbf{x}^{\prime}-\mathbf{x} ; \mathbf{m}^{\prime}, \mathbf{m}\right) f(\mathbf{x}, \mathbf{m}, t) \delta t \\
= & \int_{\Omega} \int_{\|\mathbf{m}\|=1} f(\mathbf{x}, \mathbf{m}, t) \mathbf{v}(\mathbf{x}, t) \cdot \nabla U \delta t d \mathbf{m} d \mathbf{x} \\
= & \int_{\Omega} \int_{\|\mathbf{m}\|=1} f(\mathbf{x}, \mathbf{m}, t) \mathbf{v}(\mathbf{x}, t) \cdot \nabla \mu \delta t d \mathbf{m} d \mathbf{x} .
\end{aligned}
$$

Thus we have the expression for the body force

$$
\begin{aligned}
\mathbf{F}^{e} & =-\int_{\|\mathbf{m}\|=1} \nabla \mu f(\mathbf{x}, \mathbf{m}, t) d \mathbf{m} \\
& =-\langle\nabla \mu\rangle .
\end{aligned}
$$

Note that this is not a gradient for inhomgenenous systems.

2.4. Hydrodynamic equation. The velocity field satisfies Navier-Stokes-like equation expressing conservation of mass and momentum:

$$
\begin{aligned}
\rho\left(\frac{\partial \mathbf{v}}{\partial t}+\mathbf{v} \cdot \nabla \mathbf{v}\right) & =-\nabla p+\nabla \cdot \tau+\mathbf{F}^{e} \\
\nabla \cdot \mathbf{v} & =0 .
\end{aligned}
$$

Let $\eta_{p}=\xi_{r}, \eta=\eta_{s}+\eta_{p}, \gamma=\frac{\eta_{s}}{\eta}$, we can non-dimensionalize the above system

$$
\begin{aligned}
\frac{\partial \mathbf{v}}{\partial t}+\mathbf{v} \cdot \nabla \mathbf{v}= & -\nabla p+\frac{\gamma}{R e} \triangle \mathbf{v}+\frac{1-\gamma}{2 R e} \nabla \cdot(\mathbf{D}:\langle\mathbf{m m m m}\rangle) \\
& +\frac{1-\gamma}{D e R e}\left(\nabla \cdot \tau^{e}+\mathbf{F}^{e}\right)
\end{aligned}
$$

where $R e$ is the Reynolds number. 
2.5. Energy dissipation. Let $\rho$ be the density. When the external force is neglected, the rate of energy dissipation can be calculated as

$$
\begin{aligned}
& -\frac{d}{d t}\left[\int_{\Omega} \frac{\rho}{2} \mathbf{v} \cdot \mathbf{v} d \mathbf{x}+A(f)\right] \\
= & -\int_{\Omega} \rho \frac{d \mathbf{v}}{d t} \cdot \mathbf{v} d \mathbf{x}-k_{B} T \int_{\Omega} \int_{\|\mathbf{m}\|=1} \frac{d}{d t}\left[f \ln f-f+\frac{1}{2 k_{B} T} U f\right] d \mathbf{m} d \mathbf{x} \\
= & -\int_{\Omega}\left(-\nabla p+\nabla \cdot \tau^{s}+\nabla \cdot \tau^{e}+\mathbf{F}^{e}\right) \cdot \mathbf{v} d \mathbf{x} \\
& -k_{B} T \int_{\Omega} \int_{\|\mathbf{m}\|=1} \mu \frac{d f}{d t}+\frac{1}{2 k_{B} T}\left(\frac{d U}{d t} f-U \frac{d f}{d t}\right) d \mathbf{m} d \mathbf{x} \\
= & \int_{\Omega}\left(\tau^{s}: \nabla \mathbf{v}+\tau^{e}: \nabla \mathbf{v}-\mathbf{F}^{e} \cdot \mathbf{v}\right) d \mathbf{x} \\
& -k_{B} T \int_{\Omega} \int_{\|\mathbf{m}\|=1}\left\{\frac{1}{k_{B} T} \nabla \cdot\left[D_{\|} \mathbf{m m}+D_{\perp}(I-\mathbf{m m})\right] \cdot \nabla \mu f\right. \\
& \left.+\frac{D_{r}}{k_{B} T} \mathcal{R} \cdot(f \mathcal{R} \mu)\right\}-\mu \mathcal{R} \cdot(\mathbf{m} \times \kappa \cdot \mathbf{m} f) d \mathbf{m} d \mathbf{x} \\
& -\frac{k_{B} T}{2} \int_{\Omega} d \mathbf{x} \int_{\|\mathbf{m}\|=1} d \mathbf{m} \int_{\left\|\mathbf{m}^{\prime}\right\|=1} d \mathbf{m}^{\prime} \int_{\Omega} d \mathbf{x}^{\prime} f(\mathbf{x}, \mathbf{m}, t) \\
& \left(\mathbf{v}(\mathbf{x}, t)-\mathbf{v}\left(\mathbf{x}^{\prime}, t\right)\right) \cdot \nabla B\left(\mathbf{x}-\mathbf{x}^{\prime} ; \mathbf{m}, \mathbf{m}^{\prime}\right) f\left(\mathbf{x}^{\prime}, \mathbf{m}^{\prime}, t\right) \\
= & \int_{\Omega} \tau^{s}: \nabla \mathbf{v} d \mathbf{x}+\int_{\Omega}\left\langle\nabla \mu \cdot\left[D_{\|} \mathbf{m m}+D_{\perp}\left(I-\mathbf{m m}^{\prime}\right)\right] \nabla \mu+D_{r} \mathcal{R} \mu \cdot \mathcal{R} \mu\right\rangle d \mathbf{x} \\
= & \int_{\Omega}\left[2 \eta_{s} \mathbf{D}: \mathbf{D}+\frac{\xi_{r}}{2}\left\langle\left(\mathbf{m m}: \mathbf{D}^{2}\right\rangle\right] d \mathbf{x}\right. \\
& +\int_{\Omega}\left\langle\nabla \mu \cdot\left[D_{\|} \mathbf{m m}+D_{\perp}(I-\mathbf{m m})\right] \nabla \mu\right\rangle+D_{r}\langle\mathcal{R} \mu \cdot \mathcal{R} \mu\rangle d \mathbf{x} . \\
&
\end{aligned}
$$

This is non-negative definite provided that

$$
\begin{aligned}
& \eta_{s} \geq 0, \quad \xi_{r} \geq 0, \\
& D_{\| \mathbf{m m}}+D_{\perp}(I-\mathbf{m m}) \geq 0, \\
& D_{r} \geq 0 .
\end{aligned}
$$

2.6. Equilibrium distributions. Let $f_{0}$ be the equilibrium distribution function. It satisfies the Doi-Onsager equation

$$
\mathcal{R} \cdot\left(\mathcal{R} f_{0}+f_{0} \mathcal{R} U_{0}\left[f_{0}\right]\right)=0,
$$

where $U_{0}$ is the Maier-Saupe potential, then such a potential is necessarily invariant with respect to rotations around a director $\mathbf{n} \in \mathbb{S}^{2}$, i.e., it is axially symmetric. The number of stationary solutions of the Doi-Onsager equation on the sphere depends on the intensity parameter $\alpha$. There are two critical values for $\alpha: \alpha_{1}^{*} \approx 6.731393$ and $\alpha_{2}^{*}=7.5$, the nematic phase will be appeared after $\alpha>\alpha_{1}^{*}$, the isotropic phase will lose stability after $\alpha>\alpha_{2}^{*}$. All solutions are given explicitly by

$$
f_{0}=k e^{-\eta(\mathbf{m} \cdot \mathbf{n})^{2}},
$$


where $\mathbf{n} \in \mathbb{S}^{2}$ is a parameter, $\eta=\eta(\alpha)$ and $k=\left[4 \pi \int_{0}^{1} e^{-\eta z^{2}} d z\right]^{-1}$ are determined by $\alpha$ through

$$
\frac{3 e^{-\eta}}{\int_{0}^{1} e^{-\eta z^{2}} d z}-\left(3-2 \eta+\frac{4 \eta^{2}}{\alpha}\right)=0
$$

Axial-symmetry and explicit formulas for equilibrium distribution function was established in $[10,5]$. See also the related work in [1].

The work that follows resembles the hydrodynamic limit for the kinetic theory of gases, where Deborah number plays the role of Knusden number. In particular, we will refer to $f_{0}(\mathbf{m} \cdot \mathbf{n}(\mathbf{x}))$ as local equilibrium distribution function.

3. Frank elastic energy. In classical continuum theory of liquid crystals, the elastic energy density due to the distortion of the average orientation of the liquid crystals is given by the Frank energy density:

$$
e^{F}=K_{1}(\nabla \cdot \mathbf{n})^{2}+K_{2}(\mathbf{n} \cdot \nabla \times \mathbf{n})^{2}+K_{3}(\mathbf{n} \times \nabla \times \mathbf{n})^{2}
$$

Here $\mathbf{n}$ is the so-called director field: $\mathbf{n}(\mathbf{x})$ gives the preferred orientation of the liquid crystal molecules at the position $\mathbf{x} . K_{1}, K_{2}, K_{3}$ are the elastic constants.

Several attempts have been made to evaluate these elastic constants from molecular models of the Onsager type, using either the Onsager potential [18] or the Maier-Saupe mean field potential [13].

We will make the local equilibrium approximation. In the case when the system is subject to a weak ordering field that varies slowly in space, this amounts to assuming that the response of the liquid crystal to such a field is that the preferred orientation aligns everywhere along that field, and the relative probability of a molecule at $\mathbf{x}$ having orientation $\mathbf{m}$ is $f_{0}(\mathbf{m} \cdot \mathbf{n}(\mathbf{x}))$, where $\mathbf{n}(\mathbf{x})$ is the local preferred orientation. The extra free energy due this variation of $\mathbf{n}(\mathbf{x})$ can be calculated as

$$
\begin{gathered}
E_{F}=\frac{k_{B} T}{2} \int_{\Omega} d \mathbf{x} \int_{\|\mathbf{m}\|=1} d \mathbf{m} \int_{\left\|\mathbf{m}^{\prime}\right\|=1} d \mathbf{m}^{\prime} \int_{\Omega} d \mathbf{x}^{\prime} f_{0}(\mathbf{m} \cdot \mathbf{n}(\mathbf{x})) \\
B\left(\mathbf{x}-\mathbf{x}^{\prime} ; \mathbf{m}, \mathbf{m}^{\prime}\right)\left[f_{0}\left(\mathbf{m}^{\prime} \cdot \mathbf{n}\left(\mathbf{x}^{\prime}\right)\right)-f_{0}\left(\mathbf{m}^{\prime} \cdot \mathbf{n}(\mathbf{x})\right)\right]
\end{gathered}
$$

We will now evaluate (3.2) to second order in the derivatives of $\mathbf{n}(\mathbf{x})$. The slow spatial variation of $\mathbf{n}(\mathbf{x})$ allows us to write

$$
\begin{aligned}
f_{0}\left(\mathbf{m}^{\prime} \cdot \mathbf{n}\left(\mathbf{x}^{\prime}\right)\right)-f_{0}\left(\mathbf{m}^{\prime} \cdot \mathbf{n}(\mathbf{x})\right)= & f_{0}^{\prime}\left(\mathbf{m}^{\prime} \cdot \mathbf{n}(\mathbf{x})\right)\left\{\left(\mathbf{x}^{\prime}-\mathbf{x}\right) \cdot \nabla\left(\mathbf{m}^{\prime} \cdot \mathbf{n}(\mathbf{x})\right)\right. \\
& \left.+\frac{1}{2}\left[\left(\mathbf{x}^{\prime}-\mathbf{x}\right) \cdot \nabla\right]^{2}\left(\mathbf{m}^{\prime} \cdot \mathbf{n}(\mathbf{x})\right)\right\} \\
& +\frac{1}{2} f_{0}^{\prime \prime}\left(\mathbf{m}^{\prime} \cdot \mathbf{n}(\mathbf{x})\right)\left[\left(\mathbf{x}^{\prime}-\mathbf{x}\right) \cdot \nabla\left(\mathbf{m}^{\prime} \cdot \mathbf{n}(\mathbf{x})\right)\right]+\cdots
\end{aligned}
$$

then we have

$$
\begin{aligned}
\frac{E_{F}}{k_{B} T}= & \frac{1}{2} \int_{\Omega} d \mathbf{x} \int_{\|\mathbf{m}\|=1} d \mathbf{m} \int_{\left\|\mathbf{m}^{\prime}\right\|=1} d \mathbf{m}^{\prime} \int_{\Omega} d \mathbf{x}^{\prime} f_{0}(\mathbf{m} \cdot \mathbf{n}(\mathbf{x})) \\
& B\left(\mathbf{x}-\mathbf{x}^{\prime} ; \mathbf{m}, \mathbf{m}^{\prime}\right) f_{0}^{\prime}\left(\mathbf{m}^{\prime} \cdot \mathbf{n}(\mathbf{x})\right)\left[\left(\mathbf{x}^{\prime}-\mathbf{x}\right) \cdot \nabla\left(\mathbf{m}^{\prime} \cdot \mathbf{n}(\mathbf{x})\right)\right] \\
& -\frac{1}{2} \int_{\Omega} d \mathbf{x} \int_{\|\mathbf{m}\|=1} d \mathbf{m} \int_{\left\|\mathbf{m}^{\prime}\right\|=1} d \mathbf{m}^{\prime} \int_{\Omega} d \mathbf{x}^{\prime}\left[\left(\mathbf{x}^{\prime}-\mathbf{x}\right) \cdot \nabla(\mathbf{m} \cdot \mathbf{n}(\mathbf{x}))\right] \\
& f_{0}^{\prime}(\mathbf{m} \cdot \mathbf{n}(\mathbf{x})) B\left(\mathbf{x}-\mathbf{x}^{\prime} ; \mathbf{m}, \mathbf{m}^{\prime}\right) f_{0}^{\prime}\left(\mathbf{m}^{\prime} \cdot \mathbf{n}(\mathbf{x})\right)\left[\left(\mathbf{x}^{\prime}-\mathbf{x}\right) \cdot \nabla\left(\mathbf{m}^{\prime} \cdot \mathbf{n}(\mathbf{x})\right)\right] .
\end{aligned}
$$


We assume that the first term vanishes. Otherwise the liquid crystal must be a "cholesteric" with a finite twist in the equilibrium state. The Frank elastic energy can then be written as

$$
\begin{aligned}
E_{F}= & k_{B} T \int_{\Omega} d \mathbf{x} \int_{\|\mathbf{m}\|=1} d \mathbf{m} \int_{\left\|\mathbf{m}^{\prime}\right\|=1} d \mathbf{m}^{\prime} f_{0}^{\prime}(\mathbf{m} \cdot \mathbf{n}(\mathbf{x})) f_{0}^{\prime}\left(\mathbf{m}^{\prime} \cdot \mathbf{n}(\mathbf{x})\right) \\
& B\left(\mathbf{m}, \mathbf{m}^{\prime}\right):\left[\nabla(\mathbf{m} \cdot \mathbf{n}(\mathbf{x})) \nabla\left(\mathbf{m}^{\prime} \cdot \mathbf{n}(\mathbf{x})\right)\right] \\
\simeq & \int_{\Omega}\left[K_{1}(\nabla \cdot \mathbf{n})^{2}+K_{2}(\mathbf{n} \cdot \nabla \times \mathbf{n})^{2}+K_{3}(\mathbf{n} \times \nabla \times \mathbf{n})^{2}\right] d \mathbf{x}
\end{aligned}
$$

where

$$
B\left(\mathbf{m}, \mathbf{m}^{\prime}\right)=-\frac{1}{2} \int_{\Omega} \mathbf{x x} B\left(\mathbf{x} ; \mathbf{m}, \mathbf{m}^{\prime}\right) d \mathbf{x}=\left(B_{i j}\left(\mathbf{m}, \mathbf{m}^{\prime}\right)\right) .
$$

By restricting this expression to special configurations, we obtain the Frank elastic constants:

Splay: $\mathbf{i}+y \mathbf{j}$,

$$
K_{1}=k_{B} T \int_{\|\mathbf{m}\|=1} d \mathbf{m} \int_{\left\|\mathbf{m}^{\prime}\right\|=1} d \mathbf{m}^{\prime} f_{0}^{\prime}(\mathbf{m} \cdot \mathbf{i}) f_{0}^{\prime}\left(\mathbf{m}^{\prime} \cdot \mathbf{i}\right) B_{22}\left(\mathbf{m}, \mathbf{m}^{\prime}\right)(\mathbf{m} \cdot \mathbf{j})\left(\mathbf{m}^{\prime} \cdot \mathbf{j}\right) .
$$

Twist: $\mathbf{i}+z \mathbf{j}$

$$
K_{2}=k_{B} T \int_{\|\mathbf{m}\|=1} d \mathbf{m} \int_{\left\|\mathbf{m}^{\prime}\right\|=1} d \mathbf{m}^{\prime} f_{0}^{\prime}(\mathbf{m} \cdot \mathbf{i}) f_{0}^{\prime}\left(\mathbf{m}^{\prime} \cdot \mathbf{i}\right) B_{33}\left(\mathbf{m}, \mathbf{m}^{\prime}\right)(\mathbf{m} \cdot \mathbf{j})\left(\mathbf{m}^{\prime} \cdot \mathbf{j}\right) .
$$

Bend: $\mathbf{i}+x \mathbf{j}$,

$$
K_{3}=k_{B} T \int_{\|\mathbf{m}\|=1} d \mathbf{m} \int_{\left\|\mathbf{m}^{\prime}\right\|=1} d \mathbf{m}^{\prime} f_{0}^{\prime}(\mathbf{m} \cdot \mathbf{i}) f_{0}^{\prime}\left(\mathbf{m}^{\prime} \cdot \mathbf{i}\right) B_{11}\left(\mathbf{m}, \mathbf{m}^{\prime}\right)(\mathbf{m} \cdot \mathbf{j})\left(\mathbf{m}^{\prime} \cdot \mathbf{j}\right) .
$$

4. The Ericksen-Leslie Theory. The classical Ericksen-Leslie theory describes the hydrodynamics of small liquid crystal molecules. The configuration of the liquid crystals are described by a director field $\mathbf{n}(\mathbf{x})$. The hydrodynamic equation takes the form

$$
\frac{\partial \mathbf{v}}{\partial t}+\mathbf{v} \cdot \nabla \mathbf{v}=-\nabla p+\frac{\gamma}{R e} \triangle \mathbf{v}+\frac{1-\gamma}{R e} \nabla \cdot \sigma
$$

in which stress is modelled by the phenomenological constitutive relation:

$$
\sigma=\sigma^{L}+\sigma^{E},
$$

where $\sigma^{L}$ is the viscous (Leslie) stress,

$$
\begin{aligned}
\sigma^{L}= & \alpha_{1}(\mathbf{n n}: \mathbf{D}) \mathbf{n n}+\alpha_{2} \mathbf{n N}+\alpha_{3} \mathbf{N n} \\
& +\alpha_{4} \mathbf{D}+\alpha_{5} \mathbf{n n} \cdot \mathbf{D}+\alpha_{6} \mathbf{D} \cdot \mathbf{n n} \\
\mathbf{N}= & \dot{\mathbf{n}}+\boldsymbol{\Omega} \cdot \mathbf{n}
\end{aligned}
$$

The six constants $\alpha_{1}, \cdots, \alpha_{6}$ are called the Leslie coefficients; $\sigma^{E}$ is the elastic (Ericksen) stress:

$$
\sigma^{E}=-\frac{\partial E_{F}}{\partial(\nabla \mathbf{n})} \cdot(\nabla \mathbf{n})^{T},
$$


The dynamics of the director field is governed by

$$
\begin{aligned}
& \mathbf{n} \times\left(\mathbf{h}-\gamma_{1} \mathbf{N}-\gamma_{2} \mathbf{D} \cdot \mathbf{n}\right)=0, \\
& \gamma_{1}=\alpha_{3}-\alpha_{2}, \quad \gamma_{2}=\alpha_{6}-\alpha_{5},
\end{aligned}
$$

where $\mathbf{h}$ is the molecular field

$$
\mathbf{h}=-\frac{\delta E_{F}}{\delta \mathbf{n}}=\nabla \cdot \frac{\partial E_{F}}{\partial(\nabla \mathbf{n})}-\frac{\partial E_{F}}{\partial \mathbf{n}} .
$$

As a consequence, we have the energy dissipation relation for the Ericksen-Leslie equations

$$
\begin{aligned}
& -\frac{d}{d t}\left[\int_{\Omega} \frac{R e}{2(1-\gamma)}|\mathbf{v}|^{2} d \mathbf{x}+E_{F}\right] \\
= & \frac{\gamma}{1-\gamma} \int_{\Omega}|\nabla \mathbf{v}|^{2} d \mathbf{x}+\left(\alpha_{1}+\frac{\gamma_{2}^{2}}{\gamma_{1}}\right) \int_{\Omega}|\mathbf{D}: \mathbf{n n}|^{2} d \mathbf{x} \\
& +\alpha_{4} \int_{\Omega} \mathbf{D}: \mathbf{D} d \mathbf{x}+\left(\alpha_{5}+\alpha_{6}+\lambda \gamma_{2}\right) \int_{\Omega}|\mathbf{D} \cdot \mathbf{n}|^{2} d \mathbf{x} \\
& +\frac{1}{\gamma_{1}} \int_{\Omega}|\mathbf{n} \times \mathbf{h}|^{2} d \mathbf{x} .
\end{aligned}
$$

5. The small Deborah number limit. Our aim is to derive the EricksenLeslie equation from the microscopic molecular theory represented by (2.7) and (2.8). We will consider the limit when $\varepsilon \ll 1, D e \sim O\left(\varepsilon^{2}\right)$.

5.1. Dynamics of the director field. We start with the mean-field potential:

$$
\begin{aligned}
\tilde{U}(\mathbf{x}, \mathbf{m}, t) & =U_{0}+D e U^{e} \\
U_{0} & =\int_{\Omega} \int_{\left\|\mathbf{m}^{\prime}\right\|=1} B\left(\mathbf{x}-\mathbf{x}^{\prime} ; \mathbf{m}, \mathbf{m}^{\prime}\right) f\left(\mathbf{x}, \mathbf{m}^{\prime}, t\right) d \mathbf{m}^{\prime} d \mathbf{x}^{\prime} \\
& =\int_{\left\|\mathbf{m}^{\prime}\right\|=1} B_{0}\left(\mathbf{m}, \mathbf{m}^{\prime}\right) f\left(\mathbf{x}, \mathbf{m}^{\prime}, t\right) d \mathbf{m}^{\prime} \\
U^{e} & =\frac{1}{D e} \int_{\Omega} \int_{\left\|\mathbf{m}^{\prime}\right\|=1} B\left(\mathbf{x}-\mathbf{x}^{\prime} ; \mathbf{m}, \mathbf{m}^{\prime}\right)\left[f\left(\mathbf{x}^{\prime}, \mathbf{m}^{\prime}, t\right)-f\left(\mathbf{x}, \mathbf{m}^{\prime}, t\right)\right] d \mathbf{m}^{\prime} d \mathbf{x}^{\prime}
\end{aligned}
$$

where

$$
B_{0}\left(\mathbf{m}, \mathbf{m}^{\prime}\right)=\int_{\Omega} B\left(\mathbf{x} ; \mathbf{m}, \mathbf{m}^{\prime}\right) d \mathbf{x}
$$

We make the following ansatz

$$
f(\mathbf{x}, \mathbf{m}, t)=f_{0}(\mathbf{m} \cdot \mathbf{n}(\mathbf{x}))+D e f_{1}(\mathbf{x}, \mathbf{m}, t)+o(D e),
$$

where $f_{0}$ denotes the equilibrium distribution function and $f_{1}$ is the first order perturbation. Define

$$
\Im \phi=-\mathcal{R} \cdot\left(\mathcal{R} \phi+f_{0} \mathcal{R} U_{0}[\phi]+\phi \mathcal{R} U_{0}\left[f_{0}\right]\right)
$$


then $f_{1}$ satisfies

$$
\begin{aligned}
-\Im f_{1}= & \frac{d f_{0}}{d t}-\frac{\varepsilon^{2}}{D e} \nabla \cdot\left[D_{\|}^{*} \mathbf{m m}+D_{\perp}^{*}(I-\mathbf{m m})\right]\left(\nabla f_{0}+f_{0} \nabla U_{0}\right) \\
& \left.+\mathcal{R} \cdot\left[\left(\mathbf{m} \times \kappa \cdot \mathbf{m}-\mathcal{R} U^{e}\left[f_{0}\right]\right) f_{0}\right)\right],
\end{aligned}
$$

Solvability condition gives:

$$
\begin{aligned}
& \int_{\|\mathbf{m}\|=1} \psi_{0}\left\{\frac{d f_{0}}{d t}-\frac{\varepsilon^{2}}{D e} \nabla \cdot\left[D_{\|}^{*} \mathbf{m m}+D_{\perp}^{*}(I-\mathbf{m m})\right]\left(\nabla f_{0}+f_{0} \nabla U_{0}\right)\right. \\
& \left.\left.+\mathcal{R} \cdot\left[\left(\mathbf{m} \times \kappa \cdot \mathbf{m}-\mathcal{R} U^{e}\left[f_{0}\right]\right) f_{0}\right)\right]\right\}=0,
\end{aligned}
$$

where the $\psi_{0}$ 's are non-trivial solutions of

$$
\Im^{*} \psi_{0}=0 .
$$

The solutions to this equation were characterized by Kuzuu and Doi [8]. They take the form:

$$
\psi_{0}(\mathbf{m})=\mathbf{\Theta} \cdot \mathbf{e}_{\phi} g(\theta),
$$

where $\boldsymbol{\Theta}$ is an arbitrary constant vector, $\mathbf{e}_{\phi}$ is the unit vector for the spherical coordinate $\phi$ and $g$ satisfies

$$
\frac{1}{\sin \theta} \frac{d}{d \theta}\left(\sin \theta \frac{d g}{d \theta}\right)-\frac{g}{\sin ^{2} \theta}-\frac{d U_{0}\left[f_{0}\right]}{d \theta} \frac{d g}{d \theta}=-\frac{d U_{0}\left[f_{0}\right]}{d \theta} .
$$

Let $\boldsymbol{\Omega}=\left(\kappa^{T}-\kappa\right) / 2, S_{2}=\frac{1}{2}\left(3\left\langle\cos ^{2} \theta\right\rangle-1\right)$,

$$
\lambda=\frac{2 S_{2}}{\left\langle g \frac{d U_{0}\left[f_{0}\right]}{d \theta}\right\rangle} .
$$

The terms in (5.7) can be described as follows:

$$
\begin{aligned}
& \int_{\|\mathbf{m}\|=1} \psi_{0} \frac{d f_{0}}{d t} d \mathbf{m}=\int_{\|\mathbf{m}\|=1} \psi_{0} f_{0}^{\prime}(\mathbf{m} \cdot \mathbf{n}(x)) \mathbf{m} \cdot \dot{\mathbf{n}} d \mathbf{m} \\
&=\dot{\mathbf{n}} \times \mathbf{n} \cdot \int_{\|\mathbf{m}\|=1} \psi_{0} \mathcal{R} f_{0} d \mathbf{m} \\
&=\mathbf{n} \times \dot{\mathbf{n}} \cdot\left\langle\mathcal{R} \psi_{0}\right\rangle=\boldsymbol{\Theta} \cdot(\mathbf{n} \times \dot{\mathbf{n}}) \frac{S_{2}}{\lambda} \\
& \int_{\|\mathbf{m}\|=1} \psi_{0} \mathcal{R} \cdot\left(\mathbf{m} \times \kappa \cdot \mathbf{m} f_{0}\right) d \mathbf{m}=-\int_{\|\mathbf{m}\|=1} \mathcal{R} \psi_{0}\left(\mathbf{m} \times \kappa \cdot \mathbf{m} f_{0}\right) d \mathbf{m} \\
&=\kappa:\left\langle\mathbf{m} \otimes \mathbf{m} \times \mathcal{R} \psi_{0}\right\rangle \\
&=\boldsymbol{\Theta} \cdot \mathbf{n} \times\left(S_{2} \mathbf{D} \cdot \mathbf{n}-\frac{S_{2}}{\lambda} \mathbf{\Omega} \cdot \mathbf{n}\right) \\
& \int_{\|\mathbf{m}\|=1} \psi_{0} \mathcal{R} \cdot\left(f_{0} \mathcal{R} U^{e}\right) d \mathbf{m}=\int_{\|\mathbf{m}\|=1} \mathcal{R}\left(f_{0} \mathcal{R} \psi_{0}\right) U^{e} d \mathbf{m} \\
&=\boldsymbol{\Theta} \cdot \int_{\|\mathbf{m}\|=1} \mathcal{R} f_{0} U^{e} d \mathbf{m} \\
&=\mathbf{\Theta} \cdot \mathbf{n} \times\left(-\frac{\delta\left(E_{F}\right)}{\delta \mathbf{n}}\right) \\
&=\boldsymbol{\Theta} \cdot \mathbf{n} \times \mathbf{h},
\end{aligned}
$$


where $\mathbf{h}$ is the molecular field,

$$
\mathbf{h}=-\frac{\delta\left(E_{F}\right)}{\delta \mathbf{n}},
$$

Using (2.21), and the fact that $\mu_{0}=\ln f_{0}+U_{0}$ is independent of $\mathbf{x}$, we get

$$
\int_{\|\mathbf{m}\|=1} \psi_{0} \nabla \cdot\left[D_{\|}^{*} \mathbf{m m}+D_{\perp}^{*}(I-\mathbf{m m})\right]\left(\nabla f_{0}+f_{0} \nabla U_{0}\right) d \mathbf{m}=0 .
$$

Therefore we have

$$
\boldsymbol{\Theta} \cdot(\mathbf{n} \times \dot{\mathbf{n}}) \frac{S_{2}}{\lambda}-\boldsymbol{\Theta} \cdot\left(S_{2} \mathbf{D} \cdot \mathbf{n}-\frac{S_{2}}{\lambda} \boldsymbol{\Omega} \cdot \mathbf{n}\right)=\boldsymbol{\Theta} \cdot \mathbf{n} \times \mathbf{h} .
$$

Since this is true for all $\Theta$, we have

$$
\mathbf{n} \times\left(\frac{S_{2}}{\lambda} \mathbf{N}-S_{2} \mathbf{D} \cdot \mathbf{n}\right)=\mathbf{n} \times \mathbf{h},
$$

where $\mathbf{N}=\dot{\mathbf{n}}+\boldsymbol{\Omega} \cdot \mathbf{n}$.

5.2. The viscous stress tensor. We will see that the viscous stress tensor can be expressed by the second and fourth moments of $\mathbf{m}$ only. Let

$$
Q_{2}=\left\langle\mathbf{m m}-\frac{1}{3} \mathbf{I}\right\rangle
$$

multiplying $\mathbf{m m}-\frac{1}{3} \mathbf{I}$ to both sides of (2.7) and integrating over the unit sphere, we get after some manipulation

$$
\begin{aligned}
\frac{\partial Q_{2}}{\partial t} & +\nabla \cdot\left(\mathbf{v} Q_{2}\right) \\
= & \frac{\varepsilon^{2}}{D e} \int_{\|\mathbf{m}\|=1}\left(\mathbf{m m}-\frac{1}{3} \mathbf{I}\right) \nabla \cdot\left\{\left[D_{\|}^{*} \mathbf{m m}+D_{\perp}^{*}(I-\mathbf{m m})\right] \cdot(f \nabla \mu)\right\} d \mathbf{m} \\
& +\frac{1}{D e} \int_{\|\mathbf{m}\|=1}\left(\mathbf{m m}-\frac{1}{3} \mathbf{I}\right)[\mathcal{R} \cdot(\mathcal{R} f+f \mathcal{R} \tilde{U})-D e \mathcal{R}(\mathbf{m} \times \kappa \cdot \mathbf{m} f)] d \mathbf{m}
\end{aligned}
$$

Straightforward calculation gives

$$
\begin{gathered}
\int_{\|\mathbf{m}\|=1}\left(\mathbf{m m}-\frac{1}{3} \mathbf{I}\right) \mathcal{R} \cdot(\mathcal{R} f+f \mathcal{R} \tilde{U}) d \mathbf{m}=\langle\mathbf{m} \times \mathcal{R} \mu \otimes \mathbf{m}+\mathbf{m} \otimes \mathbf{m} \times \mathcal{R} \mu\rangle \\
\int_{\|\mathbf{m}\|=1}\left(\mathbf{m m}-\frac{1}{3} \mathbf{I}\right) \mathcal{R} \cdot(\mathbf{m} \times \kappa \cdot \mathbf{m} f) d \mathbf{m},=2 \mathbf{D}:\langle\mathbf{m m m m}\rangle-\mathbf{D} \cdot\langle\mathbf{m m}\rangle+\mathbf{\Omega} \cdot\langle\mathbf{m m}\rangle \\
-\langle\mathbf{m m}\rangle \cdot \mathbf{D}-\langle\mathbf{m m}\rangle \cdot \mathbf{\Omega} .
\end{gathered}
$$

Noting that $\mu_{0}$ is independent of $\mathbf{x}$, we have

$$
\begin{aligned}
\langle\mathbf{m} \times \mathcal{R} \mu \otimes \mathbf{m}+\mathbf{m} \otimes \mathbf{m} \times \mathcal{R} \mu\rangle= & D e\left[\frac{\partial Q_{2}}{\partial t}+\nabla \cdot\left(\mathbf{v} Q_{2}\right)+2 \mathbf{D}:\langle\mathbf{m m m m}\rangle\right. \\
& -\mathbf{D} \cdot\langle\mathbf{m m}\rangle+\boldsymbol{\Omega} \cdot\langle\mathbf{m m}\rangle \\
& -\langle\mathbf{m m}\rangle \cdot \mathbf{D}-\langle\mathbf{m m}\rangle \cdot \boldsymbol{\Omega}] .
\end{aligned}
$$


The polymer stress

$$
\begin{aligned}
\tau^{p}= & \frac{D e}{2}\langle\mathbf{m m m m}\rangle: \mathbf{D}-\langle\mathbf{m} \otimes \mathbf{m} \times \mathcal{R} \mu\rangle \\
= & \frac{D e}{2}\langle\mathbf{m m m m}\rangle: \mathbf{D}-\frac{1}{2}\langle\mathbf{m} \times \mathcal{R} \mu \otimes \mathbf{m}+\mathbf{m} \otimes \mathbf{m} \times \mathcal{R} \mu\rangle \\
& -\frac{1}{2}\langle\mathbf{m} \times \mathcal{R} \mu \otimes \mathbf{m}-\mathbf{m} \otimes \mathbf{m} \times \mathcal{R} \mu\rangle .
\end{aligned}
$$

Using the symmetry of $\left\langle\mathbf{m} \otimes \mathbf{m} \times \mathcal{R} \mu_{0}\right\rangle$,

$$
\begin{aligned}
\tau^{p}= & \frac{D e}{2}\langle\mathbf{m m m m}\rangle: \mathbf{D}-\frac{D e}{2}\left[\frac{\partial Q_{2}}{\partial t}+\nabla \cdot\left(\mathbf{v} Q_{2}\right)+2 \mathbf{D}:\langle\mathbf{m m m m}\rangle\right. \\
& -\mathbf{D} \cdot\langle\mathbf{m m}\rangle+\mathbf{\Omega} \cdot\langle\mathbf{m m}\rangle-\langle\mathbf{m m}\rangle \cdot \mathbf{D}-\langle\mathbf{m m}\rangle \cdot \boldsymbol{\Omega}] \\
& -\frac{D e}{2}\left[\left\langle\mathbf{m} \otimes \mathbf{m} \mathcal{R} U^{e}\right\rangle-\left\langle\mathbf{m} \times \mathcal{R} U^{e} \otimes \mathbf{m}\right\rangle\right] .
\end{aligned}
$$

the notation of $\mathrm{mm}$ is not consistent. By definition,

$$
\begin{aligned}
\left\langle\mathbf{m} \otimes \mathbf{m} \times \mathcal{R} U^{e}\right\rangle & =\int_{\|\mathbf{m}\|=1}\left(\mathbf{m} \otimes \mathbf{m} \times \mathcal{R} U^{e}\right) f_{0} d \mathbf{m} \\
& =\int_{\|\mathbf{m}\|=1} \mathbf{m} \cdot \nabla_{\mathbf{m}} U^{e} \mathbf{m} \otimes \mathbf{m} f_{0} d \mathbf{m}-\int_{\|\mathbf{m}\|=1} \mathbf{m} \otimes \nabla_{\mathbf{m}} U^{e} f_{0} d \mathbf{m},
\end{aligned}
$$

we have

$$
\begin{aligned}
\left\langle\mathbf{m} \otimes \mathbf{m} \times \mathcal{R} U^{e}\right\rangle-\left\langle\mathbf{m} \times \mathcal{R} U^{e} \otimes \mathbf{m}\right\rangle & =\int_{\|\mathbf{m}\|=1}\left(\nabla_{\mathbf{m}} U^{e} \otimes \mathbf{m}-\mathbf{m} \otimes \nabla_{\mathbf{m}} U^{e}\right) f_{0} d \mathbf{m} \\
& =\left\langle\mathbf{m} \times U^{e} \nabla_{\mathbf{m}} f_{0}-U^{e} \nabla_{\mathbf{m}} f_{0} \otimes \mathbf{m}\right\rangle \\
& =\left\langle f_{0}^{\prime}(\mathbf{m} \cdot \mathbf{n}(\mathbf{x})) U^{e}(\mathbf{m} \otimes \mathbf{n}(\mathbf{x})-\mathbf{n}(\mathbf{x}) \otimes \mathbf{m})\right\rangle \\
& =\frac{\delta E_{F}}{\delta \mathbf{n}} \otimes \mathbf{n}(\mathbf{x})-\mathbf{n}(\mathbf{x}) \otimes \frac{\delta E_{F}}{\delta \mathbf{n}} \\
& =-\mathbf{h} \otimes \mathbf{n}(\mathbf{x})+\mathbf{n}(\mathbf{x}) \otimes \mathbf{h} .
\end{aligned}
$$

Therefore,

$$
\begin{aligned}
\tau^{p}= & \frac{D e}{2}\langle\mathbf{m m m m}\rangle: \mathbf{D}-\frac{D e}{2}\left[\frac{d Q_{2}}{d t}+2 \mathbf{D}:\langle\mathbf{m m m m}\rangle\right. \\
& -\mathbf{D} \cdot\langle\mathbf{m m}\rangle+\mathbf{\Omega} \cdot\langle\mathbf{m m}\rangle-\langle\mathbf{m m}\rangle \cdot \mathbf{D}-\langle\mathbf{m m}\rangle \cdot \mathbf{\Omega}] \\
& -\frac{D e}{2}[\mathbf{n}(\mathbf{x}) \otimes \mathbf{h}-\mathbf{h} \otimes \mathbf{n}(\mathbf{x})] .
\end{aligned}
$$

We define

$$
\begin{aligned}
Q_{4 \alpha \beta \gamma \mu}= & \left\langle m_{\alpha} m_{\beta} m_{\gamma} m_{\mu}-\frac{1}{7}\left(m_{\alpha} m_{\beta} \delta_{\gamma \mu}+m_{\gamma} m_{\mu} \delta_{\alpha \beta}+m_{\alpha} m_{\gamma} \delta_{\beta \mu}+m_{\beta} m_{\mu} \delta_{\alpha \gamma}\right.\right. \\
& \left.\left.+m_{\alpha} m_{\mu} \delta_{\beta \gamma}+m_{\beta} m_{\gamma} \delta_{\alpha \mu}\right)+\frac{1}{35}\left(\delta_{\alpha \beta} \delta_{\gamma \mu}+\delta_{\alpha \gamma} \delta_{\beta \mu}+\delta_{\alpha \mu} \delta_{\beta \gamma}\right)\right\rangle,
\end{aligned}
$$

then we obtain

$$
\begin{aligned}
\langle\mathbf{m m m m}\rangle: \mathbf{D} & =Q_{4}: \mathbf{D}+\frac{1}{7} \mathbf{D}:\langle\mathbf{m m}\rangle \mathbf{I}+\frac{2}{7}(\langle\mathbf{m m}\rangle \cdot \mathbf{D}+\mathbf{D} \cdot\langle\mathbf{m m}\rangle)-\frac{2}{35} \mathbf{D} \\
& =Q_{4}: \mathbf{D}+\frac{1}{7} \mathbf{D}:\langle\mathbf{m m}\rangle \mathbf{I}+\frac{2}{7}\left(Q_{2} \cdot \mathbf{D}+\mathbf{D} \cdot Q_{2}\right)+\frac{2}{15} \mathbf{D} .
\end{aligned}
$$


Hence the viscous stress tensor can be expressed

$$
\begin{aligned}
\tau^{p}= & \frac{D e}{2}\left[\frac{5}{7}\left(Q_{2} \cdot \mathbf{D}+\mathbf{D} \cdot Q_{2}\right)+\frac{8}{15} \mathbf{D}-Q_{4}: \mathbf{D}-\frac{1}{7} \mathbf{D}: Q_{2} \mathbf{I}\right. \\
& \left.-\boldsymbol{\Omega} \cdot Q_{2}+Q_{2} \cdot \boldsymbol{\Omega}-\frac{d Q_{2}}{d t}\right]-\frac{D e}{2}[\mathbf{n}(\mathbf{x}) \otimes \mathbf{h}-\mathbf{h} \otimes \mathbf{n}(\mathbf{x})] .
\end{aligned}
$$

In the equilibrium state, the order parameter tensors can be written as

$$
\begin{aligned}
Q_{2 \alpha \beta}= & S_{2}\left(n_{\alpha} n_{\beta}-\frac{1}{3} \delta_{\alpha \beta}\right), \\
Q_{4 \alpha \beta \gamma \mu}= & S_{4}\left[n_{\alpha} n_{\beta} n_{\gamma} n_{\mu}-\frac{1}{7}\left(n_{\alpha} n_{\beta} \delta_{\gamma \mu}+n_{\gamma} n_{\mu} \delta_{\alpha \beta}+n_{\alpha} n_{\gamma} \delta_{\beta \mu}+n_{\beta} n_{\mu} \delta_{\alpha \gamma}\right.\right. \\
& \left.\left.+n_{\alpha} n_{\mu} \delta_{\beta \gamma}+n_{\beta} n_{\gamma} \delta_{\alpha \mu}\right)+\frac{1}{35}\left(\delta_{\alpha \beta} \delta_{\gamma \mu}+\delta_{\alpha \gamma} \delta_{\beta \mu}+\delta_{\alpha \mu} \delta_{\beta \gamma}\right)\right],
\end{aligned}
$$

where

$$
S_{2}=\left\langle P_{2}(\mathbf{m} \cdot \mathbf{n})\right\rangle,
$$

and

$$
S_{4}=\left\langle P_{4}(\mathbf{m} \cdot \mathbf{n})\right\rangle
$$

and $P_{2}(\mathbf{m} \cdot \mathbf{n})$ and $P_{4}(\mathbf{m} \cdot \mathbf{n})$ are the Legendre polynomials of second- and fourthorder.

Substituting (5.15) and (5.16) into (5.14), we have

$$
\begin{aligned}
\tau^{p}= & \frac{D e}{2}\left[-S_{4}(\mathbf{D}: \mathbf{n n}) \mathbf{n n}-\frac{1}{7}(\mathbf{D}: \mathbf{n n}) \mathbf{I}-S_{2}(\mathbf{n N}+\mathbf{N n})+\left(\frac{8}{15}-\frac{10}{21} S_{2}-\frac{2}{35} S_{4}\right) \mathbf{D}\right. \\
& \left.+\left(\frac{5}{7} S_{2}+\frac{2}{7} S_{4}\right)(\mathbf{n n} \cdot \mathbf{D}+\mathbf{D} \cdot \mathbf{n n})\right]-\frac{D e}{2}(\mathbf{n h}-\mathbf{h n}) .
\end{aligned}
$$

5.3. The Leslie coefficients. Using (5.8), we have

$$
\mathbf{h}=\gamma_{1} \mathbf{N}+\gamma_{2} \mathbf{D} \cdot \mathbf{n}+C \mathbf{n}
$$

where $\gamma_{1}=\frac{S_{2}}{\lambda}, \gamma_{2}=-S_{2}, C$ is a constant. Substituting (5.18) into (5.17) we have

$$
\begin{aligned}
\tau^{p}= & \frac{D e}{2}\left[-S_{4}(\mathbf{D}: \mathbf{n n}) \mathbf{n n}-\frac{1}{7}(\mathbf{D}: \mathbf{n n}) \mathbf{I}-\left(1+\frac{1}{\lambda}\right) S_{2} \mathbf{n N}\right. \\
& -\left(1-\frac{1}{\lambda}\right) S_{2} \mathbf{N n}+\left(\frac{8}{15}-\frac{10}{21} S_{2}-\frac{2}{35} S_{4}\right) \mathbf{D} \\
& \left.+\left(\frac{5}{7} S_{2}+\frac{2}{7} S_{4}+S_{2}\right) \mathbf{n n} \cdot \mathbf{D}+\left(\frac{5}{7} S_{2}+\frac{2}{7} S_{4}-S_{2}\right) \mathbf{D} \cdot \mathbf{n n}\right] .
\end{aligned}
$$

Comparing (5.19) with (4.3), we obtain the Leslie coefficients:

$$
\begin{aligned}
& \alpha_{1}=-\frac{1}{2} S_{4}, \\
& \alpha_{2}=-\frac{1}{2}\left(1+\frac{1}{\lambda}\right) S_{2}, \\
& \alpha_{3}=-\frac{1}{2}\left(1-\frac{1}{\lambda}\right) S_{2}, \\
& \left.\alpha_{4}=\frac{1}{2}\left(\frac{8}{15}-\frac{10}{21} S_{2}-\frac{2}{35} S_{4}\right)\right), \\
& \alpha_{5}=\frac{1}{2}\left(\frac{5}{7} S_{2}+\frac{2}{7} S_{4}+S_{2}\right), \\
& \alpha_{6}=\frac{1}{2}\left(\frac{5}{7} S_{2}+\frac{2}{7} S_{4}-S_{2}\right) .
\end{aligned}
$$


We see that Parodi's relation

$$
\alpha_{2}+\alpha_{3}=\alpha_{6}-\alpha_{5}
$$

is automatically satisfied. We also have

$$
\begin{aligned}
& \gamma_{1}=\alpha_{3}-\alpha_{2}=\frac{S_{2}}{\lambda}, \\
& \gamma_{2}=\alpha_{6}-\alpha_{5}=-S_{2} .
\end{aligned}
$$

5.4. The elastic stress tensor. The body force induced by the long range (nonlocal) molecular interaction is given by

$$
\mathbf{F}^{e}=-\langle\nabla \mu\rangle=-\int_{\|\mathbf{m}\|=1}\left(\nabla_{\mathbf{x}} \mu\right) f d \mathbf{m} .
$$

Giving a virtual displacement field $\mathbf{v}(\mathbf{x})$, the work done by the body force is

$$
\int_{\Omega} \mathbf{F}^{e} \cdot \mathbf{v} d \mathbf{x}=-\int_{\Omega} \int_{\|\mathbf{m}\|=1} \mathbf{v} \cdot \nabla_{\mathbf{x}} \mu f d \mathbf{m} d \mathbf{x}
$$

Since $\mu_{0}$ is independent of $\mathbf{x}$, we have

$$
\begin{aligned}
\int_{\Omega} \mathbf{F}^{e} \cdot \mathbf{v} d \mathbf{x} & =-D e \int_{\Omega} \int_{\|\mathbf{m}\|=1} \mathbf{v} \cdot \nabla_{\mathbf{x}} U^{e} f_{0} d \mathbf{m} d \mathbf{x} \\
& =D e \int_{\Omega} \int_{\|\mathbf{m}\|=1} U^{e} \mathbf{v} \cdot \nabla_{\mathbf{x}} f_{0} d \mathbf{m} d \mathbf{x} \\
& =D e \int_{\Omega} \int_{\|\mathbf{m}\|=1} U^{e} f_{0}^{\prime} \mathbf{m} \cdot[\mathbf{v} \cdot \nabla \mathbf{n}(\mathbf{x})] d \mathbf{m} d \mathbf{x} \\
& =D e \int_{\Omega} \frac{\delta E_{F}}{\delta \mathbf{n}} \cdot[\mathbf{v} \cdot \nabla \mathbf{n}(\mathbf{x})] d \mathbf{x} .
\end{aligned}
$$

For the Frank elastic energy, we have

$$
\frac{\delta E_{F}}{\delta \mathbf{n}}=\frac{\partial E_{F}}{\partial \mathbf{n}}-\nabla \cdot \frac{\partial E_{F}}{\partial(\nabla \mathbf{n})}
$$

Therefore

$$
\begin{aligned}
\int_{\Omega} \mathbf{F}^{e} \cdot \mathbf{v} d \mathbf{x}= & D e \int_{\Omega}\left[\frac{\partial E_{F}}{\partial \mathbf{n}}-\nabla \cdot \frac{\partial E_{F}}{\partial(\nabla \mathbf{n})}\right] \cdot[\mathbf{v} \cdot \nabla \mathbf{n}(\mathbf{x})] d \mathbf{x} \\
= & D e \int_{\Omega}\left\{\frac{\partial E_{F}}{\partial \mathbf{n}} \cdot[\mathbf{v} \cdot \nabla \mathbf{n}(\mathbf{x})]+\frac{\partial E_{F}}{\partial(\nabla \mathbf{n})}:\left[\mathbf{v} \cdot \nabla^{2} \mathbf{n}(\mathbf{x})\right]\right\} d \mathbf{x} \\
& +D e \int_{\Omega}\left[\frac{\partial E_{F}}{\partial(\nabla \mathbf{n})} \cdot(\nabla \mathbf{n})^{T}\right]: \nabla \mathbf{v} d \mathbf{x} \\
= & D e \int_{\Omega} \mathbf{v} \cdot \nabla E_{F}(\mathbf{n}, \nabla \mathbf{n}) d \mathbf{x}+D e \int_{\Omega}\left[\frac{\partial E_{F}}{\partial(\nabla \mathbf{n})} \cdot(\nabla \mathbf{n})^{T}\right]: \nabla \mathbf{v} d \mathbf{x} \\
= & D e \int_{\Omega}\left[\frac{\partial E_{F}}{\partial(\nabla \mathbf{n})} \cdot(\nabla \mathbf{n})^{T}\right]: \nabla \mathbf{v} d \mathbf{x} .
\end{aligned}
$$

From this we obtain

$$
\sigma^{E}=-\frac{\partial E_{F}}{\partial(\nabla \mathbf{n})} \cdot(\nabla \mathbf{n})^{T}
$$


6. Energy dissipation in the small Deborah number limit. When the external force is neglected, the energy dissipation relation becomes

$$
\begin{aligned}
-\frac{d}{d t} E= & -\frac{d}{d t}\left[\int_{\Omega} \frac{D e R e}{2(1-\gamma)} \mathbf{v} \cdot \mathbf{v} d \mathbf{x}+A(f)\right] \\
= & -\int_{\Omega} \frac{D e R e}{1-\gamma} \frac{d \mathbf{v}}{d t} \cdot \mathbf{v} d \mathbf{x}-\int_{\Omega} \int_{\|\mathbf{m}\|=1} \mu \frac{\partial f}{\partial t} \mathbf{m} d \mathbf{x} \\
= & -\frac{D e R e}{1-\gamma} \int_{\Omega}\left(-\nabla p+\frac{\gamma}{R e} \Delta \mathbf{v}+\frac{1-\gamma}{2 R e} \nabla \cdot(\mathbf{D}:\langle\mathbf{m m m m}\rangle)\right) \cdot \mathbf{v} d \mathbf{x} \\
& +\int_{\Omega}\left(\nabla \cdot \tau^{e}+\mathbf{F}^{e}\right) \cdot \mathbf{v} d \mathbf{x}-\int_{\Omega} \int_{\|\mathbf{m}\|=1} \mu \frac{\partial f}{\partial t} d \mathbf{m} d \mathbf{x} \\
= & \int_{\Omega}\left[\frac{D e \gamma}{1-\gamma}|\nabla \mathbf{v}|^{2}+\frac{D e}{2}\left\langle(\mathbf{m m}: \mathbf{D})^{2}\right\rangle\right] d \mathbf{x} \\
& +\frac{\varepsilon^{2}}{D e} \int_{\Omega}\left\langle\nabla \mu \cdot\left[D_{\|}^{*} \mathbf{m m}+D_{\perp}^{*}(I-\mathbf{m m})\right] \nabla \mu\right\rangle+\frac{1}{D e}\langle\mathcal{R} \mu \cdot \mathcal{R} \mu\rangle d \mathbf{x},
\end{aligned}
$$

where $E$ is the total energy and

$$
A(f)=A_{0}\left(f_{0}\right)+D e E_{F} .
$$

Using (2.7), we have

$$
\begin{aligned}
& \int_{\Omega}\left[\frac{D e \gamma}{1-\gamma}|\nabla \mathbf{v}|^{2}+\frac{D e}{2}\left\langle(\mathbf{m m}: \mathbf{D})^{2}\right\rangle\right] d \mathbf{x} \\
& -\int_{\Omega} \int_{\|\mathbf{m}\|=1} \mu \cdot\left(\frac{\varepsilon^{2}}{D e} \nabla \cdot\left[D_{\|}^{*} \mathbf{m} \mathbf{m}+D_{\perp}^{*}(I-\mathbf{m m})\right] \nabla \mu+\frac{1}{D e} \mathcal{R} \cdot f \mathcal{R} \mu\right) d \mathbf{m} d \mathbf{x} \\
= & \int_{\Omega}\left[\frac{D e \gamma}{1-\gamma}|\nabla \mathbf{v}|^{2}+\frac{D e}{2}\left\langle(\mathbf{m m}: \mathbf{D})^{2}\right\rangle\right] d \mathbf{x} \\
& -\int_{\Omega} \int_{\|\mathbf{m}\|=1} \mu \cdot\left[\frac{d f}{d t}+\mathcal{R} \cdot(\mathbf{m} \times \kappa \cdot \mathbf{m} f)\right] d \mathbf{m} d \mathbf{x} \\
= & \int_{\Omega}\left[\frac{D e \gamma}{1-\gamma}|\nabla \mathbf{v}|^{2}+\frac{D e}{2}\left\langle(\mathbf{m m}: \mathbf{D})^{2}\right\rangle\right] d \mathbf{x} \\
& -\int_{\Omega} \int_{\|\mathbf{m}\|=1} \mu \cdot \frac{\partial f}{\partial t} d \mathbf{m} d \mathbf{x}+\int_{\Omega}[-\langle\mathbf{m m} \times \mathcal{R} \mu\rangle: \nabla \mathbf{v}+\langle\nabla \mu\rangle \cdot \mathbf{v}] d \mathbf{x} .
\end{aligned}
$$

Using (5.11), we obtain

$$
\begin{aligned}
-\frac{d}{d t} E & =\int_{\Omega}\left[\frac{D e \gamma}{1-\gamma}|\nabla \mathbf{v}|^{2}+\tau^{p}: \nabla \mathbf{v}+\langle\nabla \mu\rangle \cdot \mathbf{v} d x-\frac{d A(f)}{d t}\right. \\
& =\int_{\Omega}\left[\frac{D e \gamma}{1-\gamma}|\nabla \mathbf{v}|^{2}+\tau^{p}: \nabla \mathbf{v}+\langle\nabla \mu\rangle \cdot \mathbf{v} d x-D e \frac{d E_{F}}{d t} .\right.
\end{aligned}
$$


Using (5.19), (5.20) and (5.23), we have

$$
\begin{aligned}
-\frac{d}{d t} E= & D e \int_{\Omega}\left[\frac{\gamma}{1-\gamma}|\nabla \mathbf{v}|^{2}+\sigma^{L}: \nabla \mathbf{v}+\sigma^{E}: \nabla \mathbf{v}\right] d \mathbf{x} \\
& +D e \int_{\Omega}\left[\frac{\delta E_{F}}{\delta \mathbf{n}} \cdot(\mathbf{v} \cdot \nabla \mathbf{n})+\mathbf{h} \cdot \dot{\mathbf{n}}\right] d \mathbf{x} \\
= & D e \int_{\Omega}\left[\frac{\gamma}{1-\gamma}|\nabla \mathbf{v}|^{2}+\mathbf{h} \cdot \dot{\mathbf{n}}\right] d \mathbf{x}+D e \int_{\Omega}\left[\alpha_{1}(\mathbf{n n}: \mathbf{D}) \mathbf{n n}+\alpha_{2} \mathbf{n N}\right. \\
& \left.+\alpha_{3} \mathbf{N} \mathbf{n}+\alpha_{4} \mathbf{D}+\alpha_{5} \mathbf{n n} \cdot \mathbf{D}+\alpha_{6} \mathbf{D} \cdot \mathbf{n n}\right]:(\mathbf{D}+\mathbf{\Omega}) d \mathbf{x} \\
= & D e \int_{\Omega}\left[\frac{\gamma}{1-\gamma}|\nabla \mathbf{v}|^{2}+\alpha_{1}(\mathbf{n n}: \mathbf{D})^{2}+\alpha_{4} \mathbf{D}: \mathbf{D}+\left(\alpha_{5}+\alpha_{6}\right)|\mathbf{D} \cdot \mathbf{n}|^{2} d \mathbf{x}\right. \\
& +D e \int_{\Omega}\left[\gamma_{2} \mathbf{N} \cdot \mathbf{D} \cdot \mathbf{n}+\frac{1}{2}(\mathbf{h n}-\mathbf{n h}): \mathbf{\Omega}+\mathbf{h} \cdot \dot{\mathbf{n}}\right] d \mathbf{x} \\
= & D e \int_{\Omega}\left[\frac{\gamma}{1-\gamma}|\nabla \mathbf{v}|^{2}+\alpha_{1}(\mathbf{n n}: \mathbf{D})^{2}+\alpha_{4} \mathbf{D}: \mathbf{D}+\left(\alpha_{5}+\alpha_{6}\right)|\mathbf{D} \cdot \mathbf{n}|^{2} d \mathbf{x}\right. \\
& +D e \int_{\Omega}\left[\gamma_{2} \mathbf{N} \cdot \mathbf{D} \cdot \mathbf{n}+\mathbf{h} \cdot \mathbf{N}\right] d \mathbf{x} .
\end{aligned}
$$

Using (5.8), it is easy to see

$$
\begin{aligned}
\int_{\Omega}\left[\gamma_{2} \mathbf{N} \cdot \mathbf{D} \cdot \mathbf{n}+\mathbf{h} \cdot \mathbf{N}\right] d \mathbf{x}= & \int_{\Omega}\left[\gamma_{2}(\mathbf{n} \times \mathbf{N}) \cdot(\mathbf{n} \times \mathbf{D} \cdot \mathbf{n})+(\mathbf{n} \times \mathbf{h}) \cdot(\mathbf{n} \times \mathbf{N})\right] d \mathbf{x} \\
= & \frac{1}{\gamma_{1}} \int_{\Omega}|\mathbf{n} \times \mathbf{h}|^{2} d \mathbf{x}-\frac{\gamma_{2}}{\gamma_{1}} \int_{\Omega}(\mathbf{n} \times \mathbf{h}) \cdot(\mathbf{n} \times \mathbf{D} \cdot \mathbf{n}) d \mathbf{x} \\
& +\gamma_{2} \int_{\Omega}(\mathbf{n} \times \mathbf{N}) \cdot(\mathbf{n} \times \mathbf{D} \cdot \mathbf{n}) d \mathbf{x} \\
= & \frac{1}{\gamma_{1}} \int_{\Omega}|\mathbf{n} \times \mathbf{h}|^{2} d \mathbf{x}-\frac{\gamma_{2}^{2}}{\gamma_{1}} \int_{\Omega}|\mathbf{n} \times \mathbf{D} \cdot \mathbf{n}|^{2} d \mathbf{x} \\
= & \frac{1}{\gamma_{1}} \int_{\Omega}|\mathbf{n} \times \mathbf{h}|^{2} d \mathbf{x}+\lambda \gamma_{2} \int_{\Omega}\left(|\mathbf{D} \cdot \mathbf{n}|^{2}-|\mathbf{D}: \mathbf{n n}|^{2}\right) d \mathbf{x} .
\end{aligned}
$$

Finally, we get

$$
\begin{aligned}
-\frac{d}{d t} E= & D e \int_{\Omega}\left[\frac{\gamma}{1-\gamma}|\nabla \mathbf{v}|^{2}+\left(\alpha_{1}+\frac{\gamma_{2}^{2}}{\gamma_{1}}\right)|\mathbf{D}: \mathbf{n n}|^{2}+\alpha_{4} \mathbf{D}: \mathbf{D}\right. \\
& \left.+\left(\alpha_{5}+\alpha_{6}+\lambda \gamma_{2}\right)|\mathbf{D} \cdot \mathbf{n}|^{2}+\frac{1}{\gamma_{1}}|\mathbf{n} \times \mathbf{h}|^{2}\right] d \mathbf{x} .
\end{aligned}
$$

7. Conclusion. In this paper, we extended the Doi theory for homogeneous LCP flows to inhomogeneous systems. One important consequence is that a term in the form of body force resulted in this extension. In the small Deborah number limit, we recover the Ericksen-Leslie equation in which this term becomes the Ericksen stress. In addition, the Leslie coefficients are expressed in terms of three parameters: the order parameters $S_{2}$ and $S_{4}$ and the parameter $\lambda$.

Acknowledgment. Weinan E is partially supported by US ONR grant N0001401-1-0674 and National Science Fund of China for Distinguished Young Scholars 10128102 (Class B). Pingwen Zhang is partially supported by the special funds for Major State Research Projects 2005CB1704 and National Science Foundation of China 
for Distinguished Young Scholars 10225103. We thank Chun Liu and Qi Wang for helpful discussions.

\section{REFERENCES}

[1] P. Constantin, I. Kevrekidis And E.S. Titi, Asymptotic states of a Smoluchowski equation, Arch. Rat. Mech. Anal., 174 (2004), pp. 365-384.

[2] M. Doi And S. F. Edwards, The theory of Polymer Dynamics, Oxford University Press, Oxford, UK, (1986).

[3] J. ERicksen, Liquid crystals with variable degree of orientation, Arch. Rat. Mech. Anal., 113 (1991), pp. 97-120.

[4] V. Faraoni, M. Grosso, S. Crescitelli and P. L. Maffettone, The rigid-rod model for nematic polymers: an analysis of the shear flow problem, J. Rheol., 43:3 (1999), pp. 829843.

[5] I. FAtKULLin AND V. SlastiKov, Critical points of the Onsager functional on a sphere, preprint.

[6] J. J. Feng, G. L. Leal and G. Sgalari, A theory for flowing nematic polymers with orientational distortion, J. Rheol., 44:5 (2000), pp. 1085-1101.

[7] P. G. De Gennes and J. Prost, The Physics of Liquid Crystals, 2nd edition, Oxford Science Publications, 1993.

[8] N. KuzuU And M. DoI, Constitutive equation for nematic liquid crystals under weak velocity gradient derived from a molecular kinetic equation, Journal of the Physical Society of Japan, 52:10 (1983), pp. 3486-3494.

[9] T. Li, E. Vanden-Eijnden, P. Zhang And W. E, Stochastic models of polymeric liquids at small Deborah number, J. Non-Newtonian Fluid Mech., 121 (2004), pp. 117-125.

[10] H. Liu, H. Zhang and P. Zhang, Axial symmetry and Classification of Stationary solutions of Doi-Onsager equation on the sphere with Maier-Saupe potential, Comm. Math. Sci., 3 (2005), pp. 201-218.

[11] P. L. Maffettone AND S. CRescitelli, Bifurcation analysis of a molecular model for nematic polymers in shear flows, J. Non-Newtonian Fluid Mech., 59 (1995), pp. 73-91.

[12] G. Marrucci and P. L. Maffettone, Description of the liquid crystalline phase of rodlike polymers at high shear rates, Marcomolecules, 22 (1989), pp. 4076-4082.

[13] G. Marrucci and F. GReco, The elastic constants of Maier-Saupe rodlike molecule nematics, Mol. Cryst. Liq. Cryst., 206 (1991), pp. 17-30.

[14] R. NAYAK, Molecular simulation of liquid crystal polymer flow: a wavelet-finite element analysis, PhD thesis, MIT, 1998.

[15] L. Onsager, The effects of shape on the interaction of colloidal particles, Ann. N. Y. Acad. Sci., 51 (1949), pp. 627-659.

[16] A. D. REY AND T. TsujI, Orientation mode selection mechanisms for sheared nematic liquid crystalline materials, Physical Review E, 57:5 (1998), pp. 5610-5625.

[17] A. D. Rey And T. TsujI, Recent advances in theoreitcal liquid crystal rheology, Macromol. Theory Simul., 7 (1998), pp. 623-639.

[18] J. P. Straley, Frank elastic constants of the hard-rod liquid crystal, Physical Review A, 8:4 (1973), pp. 2181-83.

[19] J. K. C. Suen, Y. L. Joo And R. C. Armstrong, Molecular orientation effects in viscoelasticity, Ann. Rev. Fluid Mech., 34 (2002), pp. 417-444.

[20] Q. WANG, A hydrodynamic theory for solutions of nonhomogeneous nematic liquid crystalline polymers of different configurations, Journal of Chemical Physics, 116:20 (2002), pp. 91209136.

[21] Q. Wang, W. E, C. Liu And P. Zhang, Kinetic Theories for Flows of Nonhomogeneous Rodlike Liquid Crystalline Polymers with a Nonlocal Intermolecular Potential, Phys. Review E, Vol. 65, Art. No. 051504 (2002), Corrections: 71 (4): Art. No. 049902 (2005). 